\title{
An assessment of in-field non- destructive testing methods for detection of internal defects in standing live trees
}

Mohammad Sadegh Taskhiri, Mohammad Hadi Hafezi, Damien Holloway, Paul Turner

Mohammad Sadegh Taskhiri, Mohammad Hadi Hafezi, Damien Holloway, Paul Turner, "An assessment of in-field non-destructive testing methods for detection of internal defects in standing live trees," Proc. SPIE 10972, Health Monitoring of Structural and Biological Systems XIII, 109721F (1 April 2019); doi: $10.1117 / 12.2514270$

Event: SPIE Smart Structures + Nondestructive Evaluation, 2019, Denver, Colorado, United States 


\title{
An assessment of in-field non-destructive testing methods for detection of internal defects in standing live trees
}

\author{
Mohammad Sadegh Taskhiri ${ }^{1}$, Mohammad Hadi Hafezi ${ }^{2}$, Damien Holloway ${ }^{3}$, Paul Turner ${ }^{1}$
}

\author{
${ }^{1}$ ARC Centre for Forest Value, Discipline of ICT, College of Sciences and Engineering, \\ University of Tasmania, Hobart, Australia. \\ ${ }^{2}$ Department of Civil Engineering and Engineering Mechanics, University of Arizona, Tucson, AZ \\ 85721, USA. \\ ${ }^{3}$ Discipline of Engineering, College of Sciences and Engineering, University of Tasmania, \\ Hobart, Australia.
}

\begin{abstract}
s.
Harvesting trees that contain internal defects such as knots and cracks are neither financially nor environmentally sustainable. In hardwood plantations, it is impossible to produce sawlogs from knotty or cracked timber. The challenge is to identify internal defects in a timely and cost-effective manner prior to harvesting. The aim of this paper is to investigate non-destructive testing (NDT) methods to rapidly detect the presence of internal defects in standing live trees in plantation plots. The study highlights that whilst several methods exist, few have been actively applied in-field harvesting operations to optimise log handling and to increase transportation efficiencies. Key constraints are portability of the NDT equipment for use in-field, speed versus accuracy of measurements undertaken and the usability of different evaluation approaches for decision-support. In this paper, the field assessment involved using two non-destructive techniques, ground penetrating radar (GPR) and ultrasonics that use electromagnetic and ultrasonic sound waves respectively to penetrate the internal structure of standing trees. These assessment techniques can assist forest growers to more accurately evaluate the quality of growing stems in the field. They also open the opportunity to investigate differences across a wide selection of growing conditions and forest types to generate data that may support the generation of a software algorithm for predictive imputation of likely internal defect rates within particular forests and under particular growing conditions. The plan being to integrate this predictive imputation software into existing geographical information systems owned by industry partners to enable accurate mapping of land areas where high ratios of defects are likely to be detected to further optimise infield harvesting.
\end{abstract}

Keywords: Non-destructive testing; Eucalyptus nitens; Ultrasonic testing; GPR; Internal defects, Pruned and Unpruned log.

\section{INTRODUCTION}

Plantation hardwood estates are a potential source of various high value wood products including saw $\operatorname{logs}$ and veneer logs. Exploiting these estates has become more urgent in Tasmania where a supply shortage of these types of wood products from native hardwood estate is forecast to occur by 2027. Currently in Tasmania, Eucalyptus nitens is the dominant plantation hardwood and it is primarily processed for wood chips production and exported to the 
pulp markets. This is because the majority of the plantation stands are unthined and unpruned producing logs with significant internal defects such as knots and cracks. However, in some stands a pruning process has been implemented since the 1990s with the aim of producing logs with clear wood as a potential resource for high quality saw $\log$ as well as veneer logs. Unfortunately, in many of these pruned stands it has been discovered that numerous unpruned stems are mixed with the pruned ones. As a result, identifying the pruned stems from unpruned ones' now needs to be addressed. This very challenging task is compounded by the fact that many internal defects have been covered by bark growth during the natural pruningprocesses in unpruned trees. Due to this, there is a need to detect the internal characteristics of the standing live trees non-destructively in order to avoid supplying the defected logs to the mill which increase the transportation cost significantly.

Non-destructive testing of wood has been implemented widely to identify the log quality for various wood products. Some have evaluated the structural timber (Franca et al., 2017; Feio and Machado 2016; Ilharco et al., 2015) while other tested engineering wood products (Marhenke et al., 2018; Guan et al. 2017; Niemz and Mannes 2012). Standing tree quality assessment has been investigated by many researchers. Some used multiple sensors to detects the wood decay and the healthy level of the tree (Goh et al., 2018; Michal Kloibera et al. 2016; Ningsie Indahsuary et al. 2014; Bruce Allison 2008). Other demonstrated the usefulness of various non-invasive measurement techniques to identify the physical properties of the standing trees (Rudnicki et al., 2017; Merlo et al., 2014; Wessels et al., 2011). While these researchers mainly focused on the evaluation of the tensile strength of the log, further analysis is required to detect the internal defects of the log as any internal defects such as crack or knot make the log unsuitable for timber or veneer recovery.

In the literature several devices have been used for internal defects detection. Ultrasonic testing is one of those which has been widely used in many sectors and it gains interest by researcher in the wood sector. In a recent comparative experimental study, Espinosa et al. (2018) considered the excitation signal parameters, such as shape, temporal duration, and frequency response, and then the selection of a suitable time-of-flight determination technique. The authors compared various methods to obtain the lower uncertainty values and increase the accuracy to a correct defect identification. They concluded that the result was affected by many factors including energy transfer considering the transducer properties, the transducer-bark coupling, the bark properties and the presence of signal decay which need to be considered. In another experimental effort, Taskhiri et al (2018), used an ultrasonic testing (UT) method to identify internal defects such as cracks and knots in Eucalyptus nitens. The authors undertook line scanning using a Swiss made Ultrasound device, Pundit 200PL to compare the parameters including time of flight, velocity and amplitude of 12 billets. The result has been validated against Computed Tomography (CT) scans, and measurements of the moisture content using a thermal camera.

Computer tomography (CT) scanning is another method which has been used more in forestry science and wood industry to explore internal characteristics of tree stem. It provides more detailed picture of the log's interior constructing a 3D images, and is able to clearly show the wood layers and any internal defects such as knots and cracks. Many studies have investigated this technique for genetic trials or wood traceability from stand to the mill (Kain et al. 2016; Roussel et al. 2014; Johansson et al. 2013). In this work CT scan has been practiced for data validation and test calibration, but not a viable method for in situ testing of live trees.

Ground penetration radar (GPR) is another NDT method which is mainly used for assessing steel, concrete and stone. It has recently been implemented to scan the internal tree stem structure non-destructively . In a study, Li et al. (2018) assessed the health condition of tree trunks using GPR. The authors used the Hilbert transform algorithm and the reflection amplitudes to estimate relative dielectric constant. Reci et al. 2016 and Sbartai et al. 2014 did some laboratory measurements to identify moisture content variation in wood material. The authors tested the 
sensitivity of the electromagnetic waves reflection to the moisture to identify relations between relative permittivity and moisture content of wood. In this paper, GPR will be tested and compared with ultrasonic testing on plantation hardwood and the result will be validated through detailed images obtained from CT scan.

In this paper, the objective is to identify the internal defects in standing live trees using two widely used portable techniques, namely ultrasonic testing and electromagnetic radar, and to present the preliminary results from development of a predictive imputation model for different types of tree species. Ground Penetrating Radar (GPR) and Pundit Live Array Pro were used for the ultrasonic and electromagnetic testing, respectively, to evaluate the internal structure of standing trees for any defects, and the results were accessed against CT scan results on billets from a felled tree.

\section{EXPERIMENTS}

In this study, two billets of a 17-year-old Tasmanian eucalyptus nitens (e-nitens) have been collected from the Florentine coupein the state forest of Tasmania (Sustainable Timber Tasmania). The billets were taken from both pruned and unpruned parts of the tree. As the pruning process is within the first 6.5 meters of the tree, it can be assured that billets from any part above 6.5 meters are unpruned and have internal defects. The billets were, initially, kept in a humidity room where the moisture content was set based on the in-field test using a thermal camera. The billets were scanned by CT scanner at the Royal Hobart Hospital in order to accurately detect the location of internal defects prior to ultrasonic and GPR tests.

Non-destructive testing was conducted by electromagnetic radar and ultrasonic wave velocity measurement. The wave propagation was measured using the Pundit GPR and Pundit Live Array Pro, respectively, swiss made devices (Fig 1). For GPR a laser scanner, which is located under the device, scans the object in real time and transmits the data to an iPad. For the ultrasonic technique, the device is equipped with 8 channels which propagate the wave through the object in a echo method and every wave propagated by each channel will be received by the other 7 channels (Fig 2). This will provide a total of 56 measurements of the signal details including velocity, time of flight and amplitude. The ultrasound device (Pundit Live Array Pro) can scan an area of $20 \times 7 \mathrm{~cm}$ and the wave can be propagated to the depth of 1 meter. Thus each billet was divided to 17 to 18 parts for ultrasonic scanning in order to cover the whole area of the billet. In this experiment as the billet is circle, it has been scanned with the transducer array axis of the ultrasound device aligned with the tree's axis to ensure all parts of the device were in contact with the billet (Fig 2). 


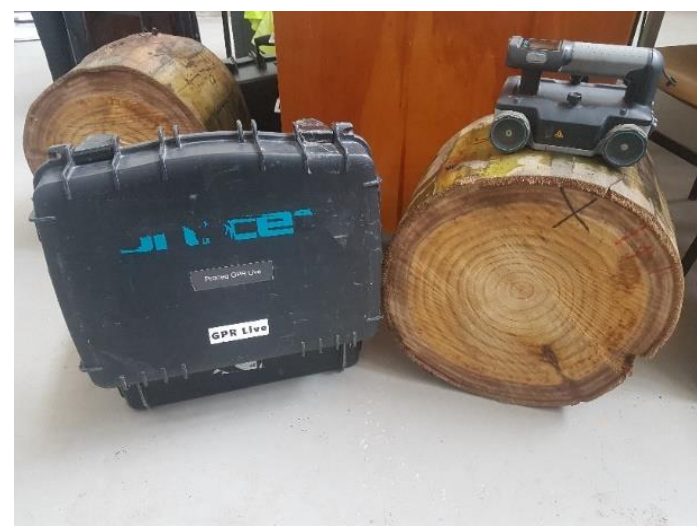

(a)



(b)

Figure 1 electromagnetic testing using GPR (a) and the ultrasonic testing using Pundit Live Array pro (b)



Figure 28 channel pulse echo measuring head principle (www.Proceq.com) 


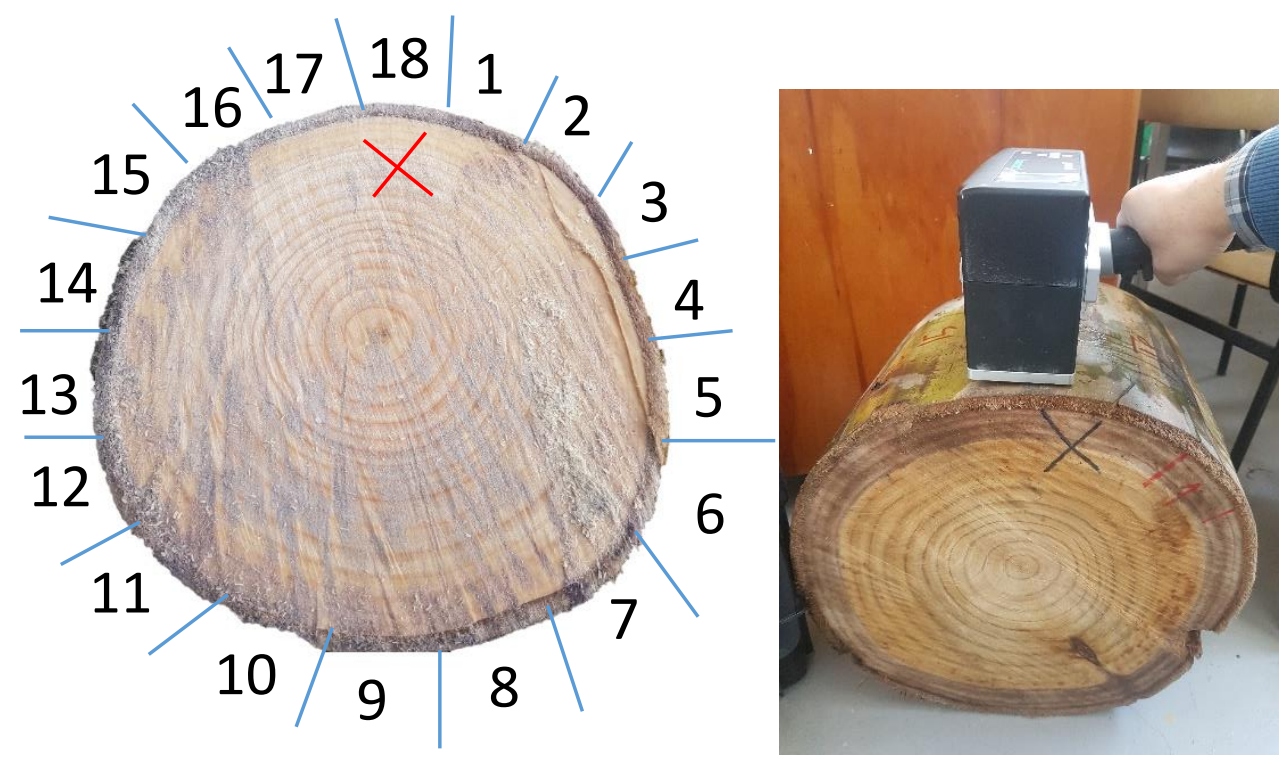

Figure 3 Ultrasonic scan of an unpruned billet using Pundit Live Array Pro

\section{INITIAL RESULTS}

As discussed in the previous section, as the first step, the logs were analysed using a medical CT scanner (Figs 4 and 5). Stacks of $512 \times 512$ pixels images were obtained. Image thickness and the interval between two images were 1.5 $\mathrm{mm}$. The pixel width (w) ranged between 0.4 and $0.8 \mathrm{~mm} /$ pixel depending on the log diameter. In total, 300 images were taken, in which each image shows the wood property at specific depth from the surface. As shown on Fig 4, two cracks were identified by the CT scan at a depth of $800 \mathrm{~mm}$ and $175 \mathrm{~mm}$ from billet's surface. The cracks had damaged the billets from the bark to the heart of the wood. This shows that this part of the tree did not undergo the pruning process. On the other hand, Fig 5 shows the pruned billet, which has no defect. The results from the CT supports the additional tests using other handheld devices such as GPR and ultrasound. It should be noted that there is a fine diagonal black line across the centre of the billets on both Fig 4, 5 which is not a defect and it is occurred during sawing process. 


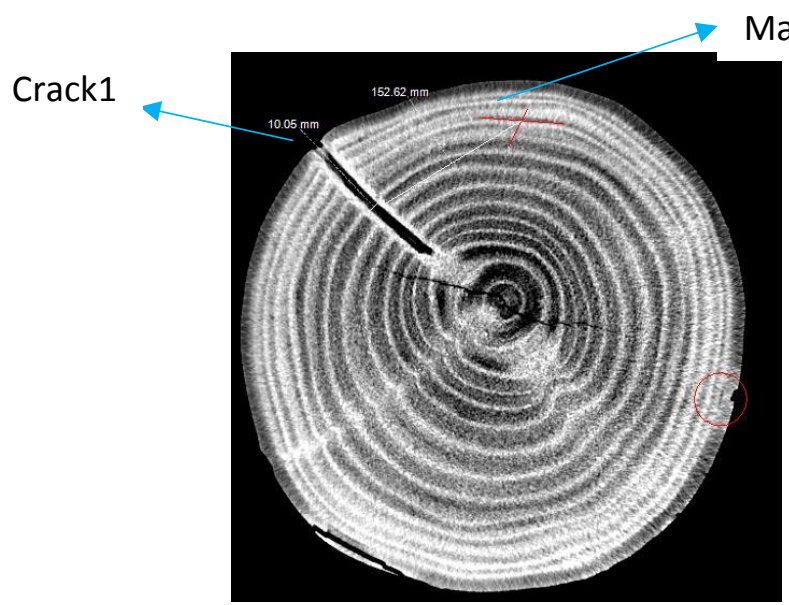

(a)

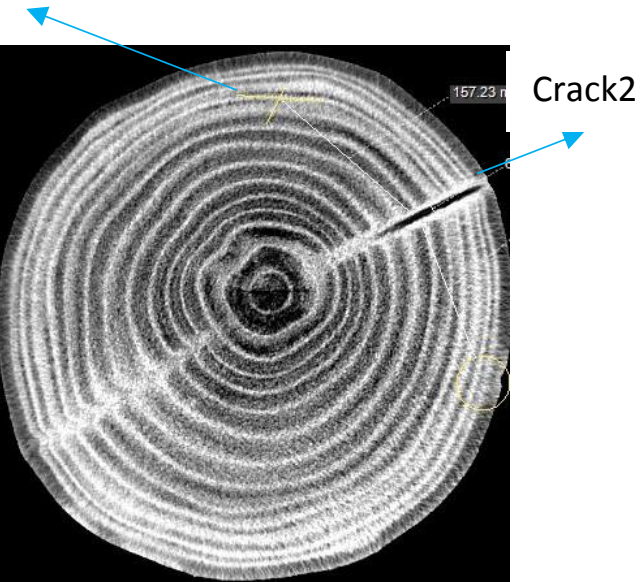

(b)

Figure 4 the detection of the internal defect at the depth of $80 \mathrm{~mm}$ (a) and $175 \mathrm{~mm}$ (b) from surface.

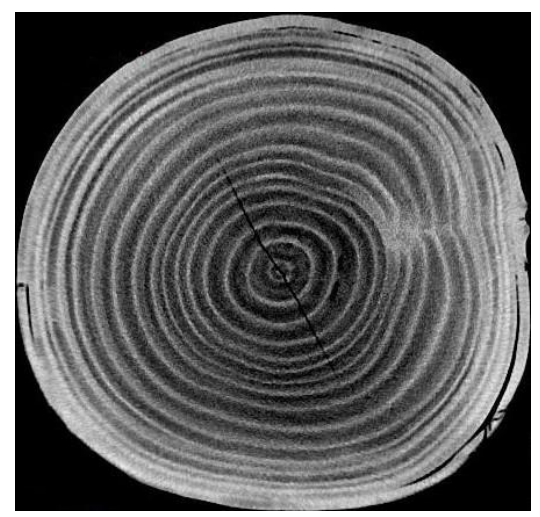

Figure $5 \mathrm{CT}$ result for a pruned billet with no internal defect

Fig 6 and 7 illustrate the result of electromagnetic and ultrasonic testing. As is evident, there is no clear result from electromagnetic testing using GPR due to very noisy result on the 2D and 3D model in Fig 4. This might be due to strong reflection of waves as they interacts with moisture content. 


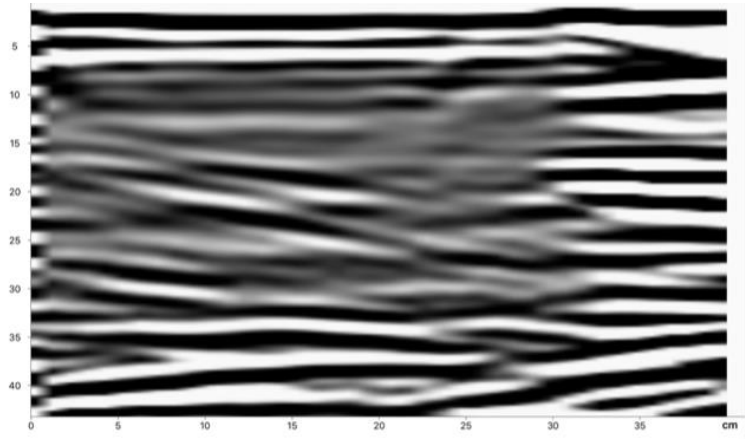

(a)

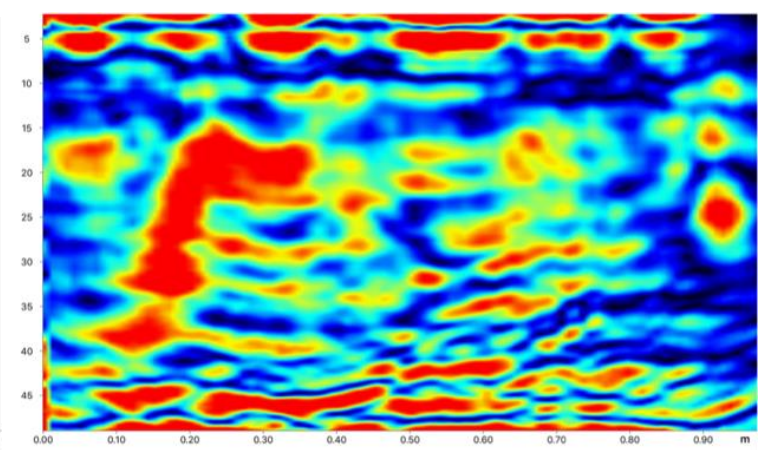

(b)



(c)

Figure 6 2D (a \& b) and 3D (c) result from GPR on unpruned billet using GPR

The result from ultrasonic testing, however, is very promising as it clearly shows the wave reflection on the internal defects (Fig 6). Fig 6 and 7 illustrate the ultrasound scan through the two billets unpruned and pruned. Both Figures comprise 18 images. This means each billet has been scanned 18 times. This is because as discussed in previous section the device can cover a maximum of $7 \mathrm{~cm}$. Thus, 18 scan are required to cover an entire billet with a circumference of $125 \mathrm{~cm}$. Due to the size of ultrasonic scan $7 \times 20 \mathrm{~cm}$, each image shows the result of the ultrasonic scan of $20 \mathrm{~cm}$ of the width of the billets.

In Fig 7, the reflections of waves are shown via red and blue colours. The blue colours represent the clear wood and the red colour demonstrates the potential existence of internal defects. It should be noted that the red colour does not necessarily identify only internal defects as wave reflections from high moisture might also be indicated by the red colour. In both Figs 7 and 8, the first image is the first ultrasound scan at the location of the marker in Fig 4 . This means that cracks 1 and 2 of the unpruned billet (Fig 4) are shown in images 3 and 15, respectively, of the corresponding ultrasonic scan (Fig 7). Both images 3 and 15 shows red colours and this begins at the previous image and continued to the next two images. There are also red colours on other images such as image 11 or 8 , however, those are not connected with the red colours in the next or previous image. Thus the red colours on images 8 or 11 indicate wave reflection from moisture and not internal defects. This has been validated through CT result (Fig 4). Fig 8 displays a completely blue colour throughout all images which demonstrates the lack of any internal defects.

Although the results from the ultrasound device, Pundit Array Live Pro, might be less conclusive without validation through the CT scan, the results show much promise towards non-destructively identifying internal defects such as knots or cracks within standing live trees. However, further research is required to analyse the data to develop a fully reliable predictive model for the NDT evaluation using ultrasonic scanning. 

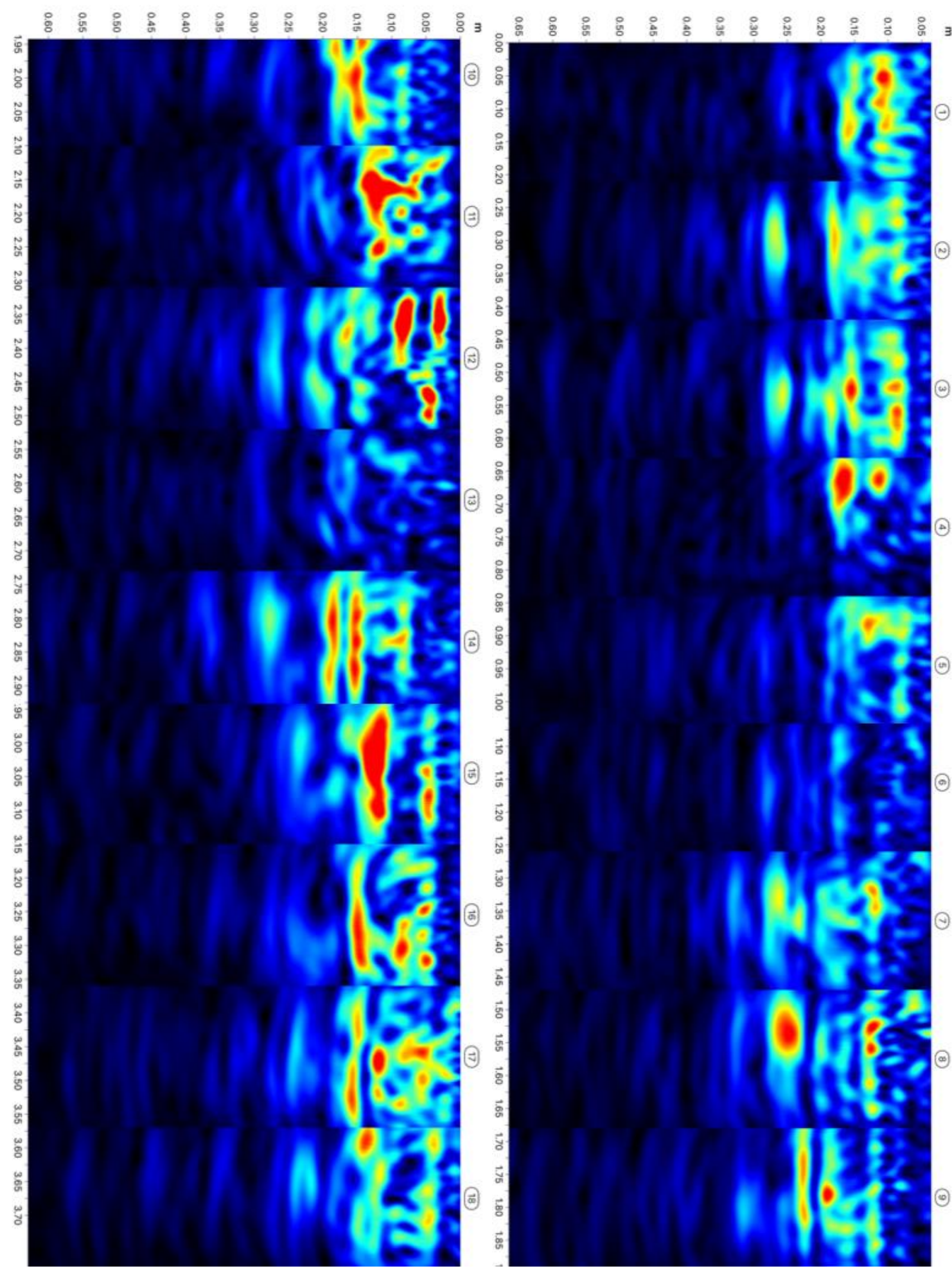

Figure 7 Ultrasonic testing for the unpruned billet with internal defects (radial direction shown on vertical axis, each of the image shows results over a 20 degree sector centred around the crack shown in Figure 4 at different heights in the billet) 



Figure 8 Ultrasonic testing for a pruned billet with no internal defects (radial direction shown on vertical axis, each of the image shows result over a 18 degree sector centred around the billet shown in Figure 5 at different heights in the billet)

Proc. of SPIE Vol. 10972 109721F-9 


\section{DISCUSSION and CONCLUSION}

Clear wood and defective (i.e. knotted or cracked) samples were analysed by the two different portable devices and compared to CT scan. As mentioned, there were much more obvious differences between clear and defective samples in the ultrasound test results then in the GPR results, suggesting good promise for the detection of defects by the former method. However, the nature of the defects was not immediately obvious from visual inspection of the output, and some experience was required to identify them. Furthermore, in addition to easier and more reliable identification of defects, it would be useful to quantify parameters such as the number, type, location, orientation and size of the defects.

It is proposed that a robust and reliable defect characterisation would best be accomplished using machine learning methods. This would require extraction of the raw data from the Pundit Live Array Pro and reformatting to arrange it in a three-dimensional model, prior to applying the machine learning algorithms. A significantly larger dataset would then be required for training and validation of the model

In this project two devices have been tested to evaluate the internal defect in standing live trees non-destructively. Although, there is no significant result from electromagnetic testing using GPR, ultrasonic testing using Pundit Array Live Pro demonstrated very promising result to detect and locate the internal defects. As the next steps, a trial will be setup to test this device on 30 trees. The collected data will be used in developing the imputation model.

\section{ACKNOWLEDGEMENTS}

The authors acknowledge the support of the Australian Research Council Industrial Transformation Training Hub 'The Centre for Forest Value' http://www.utas.edu.au/arc-forest-value.

\section{REFERENCES}

Espinosa, L., Bacca, J., Prieto, F., Lasaygues, P., \& Brancheriau, L. (2018). Accuracy on the time-of-flight estimation for ultrasonic waves applied to non-destructive evaluation of standing trees: a comparative experimental study. Acta Acustica united with Acustica, 104(3), 429-439.

Feio, A., \& Machado, J. S. (2015). In-situ assessment of timber structural members: Combining information from visual strength grading and NDT/SDT methods-A review. Construction and Building Materials, 101, 1157-1165.

França, T.S.F.A., França, F.J.N., \& Seale, R.D. (2017). Effect of Visual Characteristics on Stiffness and Strength in Southern Pine No. $22 \times 8$ Lumber. 20th International Nondestructive Testing and Evaluation of Wood Symposium.

Goh, C. L., Rahim, R. A., Rahiman, M. H. F., Talib, M. T. M., \& Tee, Z. C. (2018). Sensing wood decay in standing trees: A review. Sensors and Actuators A: Physical, 269, 276-282.

Guan, C., Zhang, H., Wang, X., Miao, H., Zhou, L., \& Liu, F. (2017). Experimental and Theoretical Modal Analysis of Full-Sized Wood Composite Panels Supported on Four Nodes. Materials, 10(6), 683. 
Ilharco, T., Lechner, T., \& Nowak, T. (2015). Assessment of timber floors by means of non-destructive testing methods. Construction and Building Materials, 101, 1206-1214.

Johansson, E., Johansson, D., Skog, J., \& Fredriksson, M. (2013). Automated knot detection for high speed computed tomography on Pinus sylvestris L. and Picea abies (L.) Karst. using ellipse fitting in concentric surfaces. Computers and electronics in agriculture, 96, 238-245.

Kain, G., Charwat-Pessler, J., Barbu, M. C., Plank, B., Richter, K., \& Petutschnigg, A. (2016). Analyzing wood bark insulation board structure using X-ray computed tomography and modeling its thermal conductivity by means of finite difference method. Journal of Composite Materials, 50(6), 795-806.

Li, W., Wen, J., Xiao, Z., \& Xu, S. (2018). Application of ground-penetrating radar for detecting internal anomalies in tree trunks with irregular contours. Sensors, 18(2), 649.

Marhenke, T., Leibniz, T., Hasener, J., \& Wallaschek, J. (2017). Determination of Mechanical Properties of Wood Based Materials using Modal Updating. 20th International Nondestructive Testing and Evaluation of Wood Symposium.

Merlo, E., Gonzalez, J. A., Santaclara, O., \& Riesco, G. (2014). Modelling modulus of elasticity of Pinus pinaster Ait. in northwestern Spain with standing tree acousting measurements, tree, stand and site variables. Forest systems, 23(1), 153-166.

Niemz, P., \& Mannes, D. (2012). Non-destructive testing of wood and wood-based materials. Journal of Cultural Heritage, 13(3), S26-S34.

Reci, H., Maï, T. C., Sbartaï, Z. M., Pajewski, L., \& Kiri, E. (2016). Non-destructive evaluation of moisture content in wood using ground-penetrating radar. Geoscientific Instrumentation, Methods and Data Systems, 5(2), 575-581.

Roussel, J. R., Mothe, F., Krähenbühl, A., Kerautret, B., Debled-Rennesson, I., \& Longuetaud, F. (2014). Automatic knot segmentation in CT images of wet softwood logs using a tangential approach. Computers and electronics in agriculture, 104, 46-56.

Sbartaï, Z. M., Maï, C., Bos, F., Razafindratsima, S., \& Demontoux, F. (2014, June). Non Destructive Evaluation of Timber Structures Using GPR technique. In congrès 15th International Conference on Ground Penetrating Radar (GPR 2014).

Taskhiri, M. S., Hafezi, M. H., Turner, P., \& Kundu, T. (2018, March). Non-destructive evaluation of a plantation eucalyptus. In Health Monitoring of Structural and Biological Systems XII(Vol. 10600, p. 1060023). International Society for Optics and Photonics.

Wessels, C. B., Malan, F. S., \& Rypstra, T. (2011). A review of measurement methods used on standing trees for the prediction of some mechanical properties of timber. European Journal of Forest Research, 130(6), 881-893. 